\title{
Article
}

\section{Combing oncolytic adenovirus expressing Beclin-1 with chemotherapy agent doxorubicin synergistically enhances cytotoxicity in human CML cells in vitro}

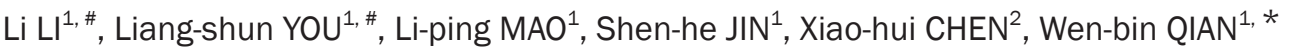 \\ ${ }^{1}$ Department of Hematology, the First Affiliated Hospital, College of Medicine, Zhejiang University, Hangzhou 310003, China; \\ ${ }^{2}$ Department of Hematology, the Affiliated Hospital of Hangzhou Normal University, Hangzhou 310015, China
}

\begin{abstract}
Cancer virotherapy provides a new strategy to treat cancer that can directly kill cancer cells by oncolysis. Insertion of therapeutic genes into the genome of a modified adenovirus, thereby creating a so-called gene-virotherapy that shares the advantages of gene therapy and virotherapy. In this study we investigated whether a strategy that combines the oncolytic effects of an adenoviral vector with the simultaneous expression of the autophagy gene Beclin-1 offered a therapeutic advantage for chronic myeloid leukemia (CML) cells with resistance to chemotherapy and evaluated the synergistic effects of SG511-BECN and doxorubicin (Dox) in human CML cells in vitro. Oncolytic virus SG511-BECN was constructed through introducing the Beclin-1 gene into the oncolytic adenoviral backbone. SG511BECN displayed significantly improved antileukemia activity on multidrug-resistant CML cell line K562/A02, which was mediated via induction of autophagic cell death. Furthermore, Dox could synergize with SG511-BECN to kill the CML cells by improving the infectious efficiency of the oncolytic adenovirus without causing significant damage to normal human mononuclear cells. The results demonstrate that targeting the autophagic cell death pathway and combination of a chemotherapy agent with oncolytic adenovirus may be a novel strategy for the treatment of leukemia with chemotherapy resistance.
\end{abstract}

Keywords: chronic myeloid leukemia; multidrug-resistant; K562/A02 cell line; doxorubicin; oncolytic adenovirus; Beclin-1; autophagy; apoptosis; synergy

Acta Pharmacologica Sinica (2018) 39: 251-260; doi: 10.1038/aps.2017.100; published online 14 Sep 2017

\section{Introduction}

Cancer virotherapy provides a new strategy to treat cancer as biotherapeutic agents that lack cross-resistance with currently available treatments ${ }^{[1-2]}$ and represents a novel approach for gene therapy ${ }^{[3-4]}$. Results from preclinical studies with several viral types are promising, among which conditionally replicating adenoviruses (CRAds) are attractive because of several of their attributes, including lytic replication, efficient genome transfer, and excellent patient safety in clinical trials ${ }^{[4-5]}$.

To enhance the therapeutic index of CRAds, two main strategies are currently being used to engineer CRAds to make them more selective and cytotoxic to tumor cells. The first approach is the creation of chimeric vectors, where the whole fiber or only the knob region is replaced with that of another serotype of adenovirus (Ad), leading to decreased hepatotoxicity fol-

\footnotetext{
\# These authors contributed equally to this work.

*To whom correspondence should be addressed.

E-mail qianwb@zju.edu.cn

Received 2017-03-07 Accepted 2017-07-09
}

lowing virus administration attributed to less liver tropism, and increased infectivity of the target tumor by coxsackie adenovirus receptor (CAR)-independent transduction ${ }^{[6-8]}$. Another strategy is based on the insertion of therapeutic genes into the genome of a modified CRAd, thereby creating a socalled gene-virotherapy. Gene-virotherapy shares the advantages of gene therapy and virotherapy, which not only can directly kill cancer cells by oncolysis but also augment the copies of therapeutic genes by replication of the virus, resulting in longer transgene expression within tumors and potent activity against cancers ${ }^{[9-10]}$. We previously generated a series of E1B55K-deleted CRAds armed with different pro-apoptotic genes, such as tumor necrosis factor-related apoptosis-inducing ligand (TRAIL), p53, and interleukin-24, and demonstrated that the combination of pro-apoptotic or tumor suppressor genes and viral oncolysis yielded an additive cytotoxic effect on cancer cells. These viruses also proved more effective than the unarmed control vector at suppressing tumor growth in vivo and prolonged survival in tumor or leukemia models ${ }^{[8,11-12]}$.

Cancer is a complex disorder associated with defects in 
multiple signaling pathways that confer resistance to apoptosis, suggesting the need for other innovative strategies ${ }^{[12-13]}$. Recent studies have demonstrated that autophagic cell death may serve as a novel way to eliminate tumor cells with defective apoptosis ${ }^{[14-18]}$. Therefore, we reasoned that arming CRAds with the genes inducing autophagic cell death could effectively kill cancer cells, especially cancer cells resistant to apoptosis, and represent an attractive prospect. Indeed, in our previous study ${ }^{[19]}$, we have demonstrated that combined Beclin-1 gene therapy that induces autophagic cell death and the SG511 vector (a new Ad5/11 fiber chimeric CRAd) showed enhanced anti-leukemia activities.

Current therapeutic approaches for CML mainly include Bcr-Abl-targeted drug or cytotoxic agents and stem cell transplantation ${ }^{[20]}$. Although the efficacy of Bcr-Abl tyrosine kinase inhibitors (TKIs) is undoubtedly for CML therapy, resistance remains a challenge ${ }^{[21]}$. Causes of the drug resistance include P-glycoprotein (P-gp) up-regulation, Abl kinase domain mutations, and Bcr-Abl overexpression ${ }^{[22]}$. Thus, novel approaches must be adopted to reverse drug resistance and enhance the therapeutic efficacy.

In this study, we combined the Beclin-1 gene therapy that induces autophagic cell death with the SG511 vector. The results showed that overexpression of Beclin-1 significantly enhanced the killing effect of the virus in multidrug-resistant CML cells, in which the cytotoxic activity of the parental virus without the Beclin-1 gene was weak overall. Next, we evaluated whether the combination treatment of SG511-BENC plus Dox performed robust synergistic killing in CML cells. We further studied the mechanism of enhanced cytotoxicity induced by the combination therapy focusing on the alteration of the virus infectious efficiency and autophagy/apoptosisassociated proteins, suggesting targeting the autophagic cell death pathway may be a novel strategy for the treatment of leukemia with chemotherapy resistance.

\section{Materials and methods}

\section{Normal bone marrow samples, cell lines and reagents}

Normal bone marrow samples from healthy volunteers were obtained after informed consent and with the approval of the Ethics Committee of the First Affiliated Hospital of Zhejiang University (Hangzhou, China). Bone marrow mononuclear cells (MNCs) were isolated by gradient centrifugation using lymphocyte cell separation medium. The human CML cell line K562 was obtained from the American Type Culture Collection (ATCC, Rockville, MD, USA). K562/A02, the doxorubicin-resistant CML cell line, was kindly provided by the Institute of Hematology, Chinese Academy of Medical Sciences (Tianjin, China). The cell lines were authenticated by comparing the immunophenotype, the karyotype, and molecular markers. All the cell lines were used within 6 months after documentation and were cultured as previously described ${ }^{[10]}$. Dox was purchased from Sigma (St Louis, MO, USA). All primary antibodies used in this study were purchased from Cell Signaling Technology (Beverly, MA, USA), except for antiBeclin-1 (Novus Biologicals), anti-LC3 (Novus Biologicals), and anti-actin (Santa Cruz Biotechnology, Santa Cruz, CA, USA).

\section{Construction of recombinant viruses}

The complete cDNA sequence of the Beclin- 1 gene was amplified by quantitative real-time-PCR (qPCR) using the upstream primer (5'-CCG GAA TTC ACC ATG GAA GGG TCT AAG ACGTCC AAC-3') and downstream primer (5'-ACG CGTCGA CTT ATC ATT TGT TAT AAA ATT GTG AGG-3'). The synthetic DNA was released with EcoRI and SalI (New England Biolabs, Beverley, MA, USA) and ligated into plasmid pENTR12 to generate pENTR12-BECN. After sequence confirmation, the pENTR12-BECN construct was then recombined using the LR reaction (Invitrogen, Carlsbad, CA, USA) into the pPE11 to form pPE11-BECN. pPE11 is an adenovirus packaging plasmid constructed by replacing the Ad5 fiber with the Ad5/11 chimeric fiber $^{[3]}$. Finally, pPE11-BECN was transfected into HEK293 cells using Lipofectamine 2000 transfection reagent (Invitrogen) together with plasmid pSG500 $0^{[2]}$. The obtained CRAds was named SG511-BECN. The enhanced green florescence protein (GFP) gene was cloned into SG511 to construct SG511-GFP as previously described ${ }^{[10]}$.

\section{Cell proliferation assay}

Cell proliferation was determined by the MTT-based cytotoxicity assay (Sigma), as previously described ${ }^{[16]}$. Briefly, cells were treated with various concentrations of Dox, SG511, or SG511-BECN for $48 \mathrm{~h}$. Upon termination, the medium was replaced with fresh medium containing $0.5 \mathrm{mg} / \mathrm{mL}$ MTT. The cells were incubated for an additional $4 \mathrm{~h}$ at $37^{\circ} \mathrm{C}$. Following removal of the medium and MTT, DMSO $(200 \mu \mathrm{L})$ was added to each well, and the absorbance was measured at $570 \mathrm{~nm}$ by spectrophotometry.

\section{Flow cytometric analysis}

After treatment with SG511-BECN and/or Dox for $48 \mathrm{~h}$, cells were harvested and washed with PBS buffer containing 5 $\mathrm{mmol} / \mathrm{L}$ EDTA and then were incubated at $37^{\circ} \mathrm{C}$ for $30 \mathrm{~min}$. Cell death was determined by staining cells with annexin V-FITC and propidium iodide (PI) using the annexin V-FITC apoptosis detection kit (BD Pharmingen, San Diego, CA, USA) and then analyzed using a FACScan flow cytometer and CELL Quest software (Becton Dickinson, Franklin Lakes, NJ, USA).

\section{Detection of autophagosome formation with acridine orange}

To detect the presence of AVOs, leukemic cells were treated with SG511 or SG511-BECN. After $48 \mathrm{~h}$, the cells were stained with the vital dye acridine orange $(1 \mu \mathrm{g} / \mathrm{mL}$; Molecular Probes, Eugene, OR, USA) and then were examined immediately by florescence microscopy (IX71; Olympus, Tokyo, Japan) with a red filter (excitation, $560 \mathrm{~nm}$; emission, $645 \mathrm{~nm}$ ).

\section{Autophagosome staining with GFP-LC3}

K562 cells stably expressing GFP-LC3 were established as previously described ${ }^{[12]}$. The cells were treated with SG511 
or SG511-BECN at an MOI of 40 for $48 \mathrm{~h}$. The florescence of GFP-LC3 was viewed under a florescent microscope, and photographs were taken. The cells with GFP-LC3 vacuoles (autophagosomes) were counted by image analysis using Image J software (National Institutes of Health, Bethesda, MD, USA).

\section{Western blot analyses}

Western blot analyses were performed as previously described ${ }^{[12]}$. Briefly, equal amounts of total cell lysates were separated on sodium dodecyl-sulfate (SDS)-polyacrylamide gels containing $8 \%-12 \%$ acrylamide gradients and then were transferred to polyvinylidene difluoride membranes (Millipore, Bedford, MA, USA). Non-specific binding was blocked with $10 \mathrm{mmol} / \mathrm{L}$ Tris-HCL buffered saline plus $0.05 \%$ Tween20 containing $5 \%$ skimmed milk powder for $1 \mathrm{~h}$ at room temperature. Membranes were incubated with the primary antibody overnight at $4^{\circ} \mathrm{C}$, followed by incubation with secondary antibodies conjugated with the fluorescent dyes for $2 \mathrm{~h}$ at room temperature. After washing three times, the membranes were incubated with anti-rabbit/mouse IgG conjugated to horseradish peroxidase. The results were visualized with the ECL detecting kit. The primary antibodies used here were as follows: caspase-8/-9/-3, LC3, BECN-1 and $\beta$-actin.

\section{Transmission electron microscopy}

CML cells were harvested after treatment with the oncolytic viruses or Dox and then were fixed with $2.5 \%$ phosphatebuffered glutaraldehyde, followed by postfixation in $1 \%$ phosphate-buffered osmium peroxide and embedding in Spurr's resin. The ultrathin sections $(0.12 \mu \mathrm{m})$ were cut and then were double stained with uranyl acetate and lead citrate. Representative areas were chosen and viewed with a Philips TECNA10 TEM at an accelerating voltage of $80 \mathrm{kV}$.

\section{Statistical analysis}

Statistical analysis of the experimental results was conducted by Student's $t$-test. A $P$-value $<0.05$ was considered to indicate a statistically significant difference. Synergisms in the combination treatments were analyzed using CalcuSyn software (Biosoft, Cambridge, UK). According to this method, synergy was expressed as $\log 10$ (CI) vs fraction affected, and $\log 10$ (CI) $<0$ indicates synergy; $\log 10(\mathrm{CI})=0$ indicates an additive effect; and $\log 10(\mathrm{CI})>0$ indicates antagonism.

\section{Results}

Oncolytic virus SG511-BECN mediates multidrug-resistant cell killing in an apoptotic-independent manner

It has been shown that SG511-BECN could induce leukemia cell death in our previous studies ${ }^{[19]}$. We first examined the effect of SG511-BECN on CML cell lines K562 and K562/A02 (a doxorubicin-resistant cell line). As shown in Figure 1B and Figure 1C, CML cells were exposed to $40 \mathrm{MOI}$ of SG511-BECN virus for $48 \mathrm{~h}$, and the viability of K562 and K562/ A02 cells was reduced by $39.33 \%$ and $54.67 \%$, respectively, indicating that K562/ A02 cells were more sensitive to SG511-BECN.
However, the doses of Dox that inhibited 50\% proliferation $\left(\mathrm{IC}_{50}\right)$ at $48 \mathrm{~h}$ in the two types of cells were approximately 0.17 and $2.51 \mu \mathrm{mol} / \mathrm{L}$, respectively. These data indicate that SG511-BECN is a potential oncolytic virus that will kill chemotherapy-sensitive and -resistant CML cells.

To further clarify whether the anti-proliferative activity of SG511-BECN was related to induction of apoptosis, Annexin V-FITC and PI double staining was performed. Treatment of K562 and K562/A02 cells with 40 MOI of SG511-BECN for $48 \mathrm{~h}$ resulted in $11.6 \%$ and $10.9 \%$ apoptotic (annexin $\mathrm{V}^{+}$and $\mathrm{PI}^{+}$) cells, respectively (Figure 2A). There was no significant difference between the response to SG511-BECN and SG511. While $0.2 \mu \mathrm{mol} / \mathrm{L}$ Dox induced $20.2 \%$ of apoptotic in K562 cells, Dox cytotoxicity against K562/ A02 was not significantly induced by $2 \mu \mathrm{mol} / \mathrm{L}$ Dox, suggesting that the killing of CML cells by SG511-BECN bypassed apoptotic pathway.

To further confirm that the anti-proliferative effect of SG511BECN occurred in an apoptotic-independent way, apoptotic signaling elements were analyzed by Western blotting. As shown in Figure 2B, treating CML cells with 40 MOI of SG511BECN did not significantly increase the levels of cleaved forms of caspase-9, -3, and PARP compared with treating K562 cells with $0.2 \mu \mathrm{mol} / \mathrm{L}$ Dox.

Taken together, these data suggest that the killing of chemotherapy-sensitive and -resistant CML cells by SG511-BECN occurs via an apoptotic-independent pathway.

\section{SG511-BECN virus induces autophagic cell death in CML cells}

To investigate whether the infection of CML cells with SG511BECN can produce Beclin-1 protein, K562 and K562/ A02 cells were exposed to $40 \mathrm{MOI}$ of virus for $48 \mathrm{~h}$ and were subjected to Western blot analysis. Beclin-1 protein showed increased expression obviously in the SG511-BECN group but not in the SG511 and Dox treatment groups.

Autophagy is characterized by the formation of AVOs and the location of microtubule-associated protein LC3 on autophagosomes ${ }^{[15]}$. Thus, we detected autophagy by measuring the following: (1) conversion of LC3-I to LC3-II by Western blotting; (2) formation of GFP-LC3-labeled vacuoles by transfection and fluorescence microscopy; (3) formation of AVOs by florescence microscopy; and (4) electron microscopy (EM) of AVOs. As expected, sharp up-regulation of LC3-II was observed in SG511-BECN-treated cells but not in PBSor Dox-treated cells; however, weak up-regulation of LC3-II was observed in cells infected with SG511 (Figure 3A). Furthermore, SG511-BECN also resulted in the significant formation of GFP-LC3-labeled vacuoles (Figure 3B) and induced AVO formation in CML cells over a 48-h time course (Figure 3C), whereas the SG511 vector did not (data not shown). In agreement with those results, EM showed many autophagic vacuoles in the cells treated with SG511-BECN (Figure 3D). By contrast, these vacuoles were not seen in the PBS-, Dox- or SG511-treated cells (data not shown).

Based on the overexpression of Beclin-1 and induced autophagy in SG511-BECN-infected cells, while activation of the caspase pathway was not observed, we conclude that 

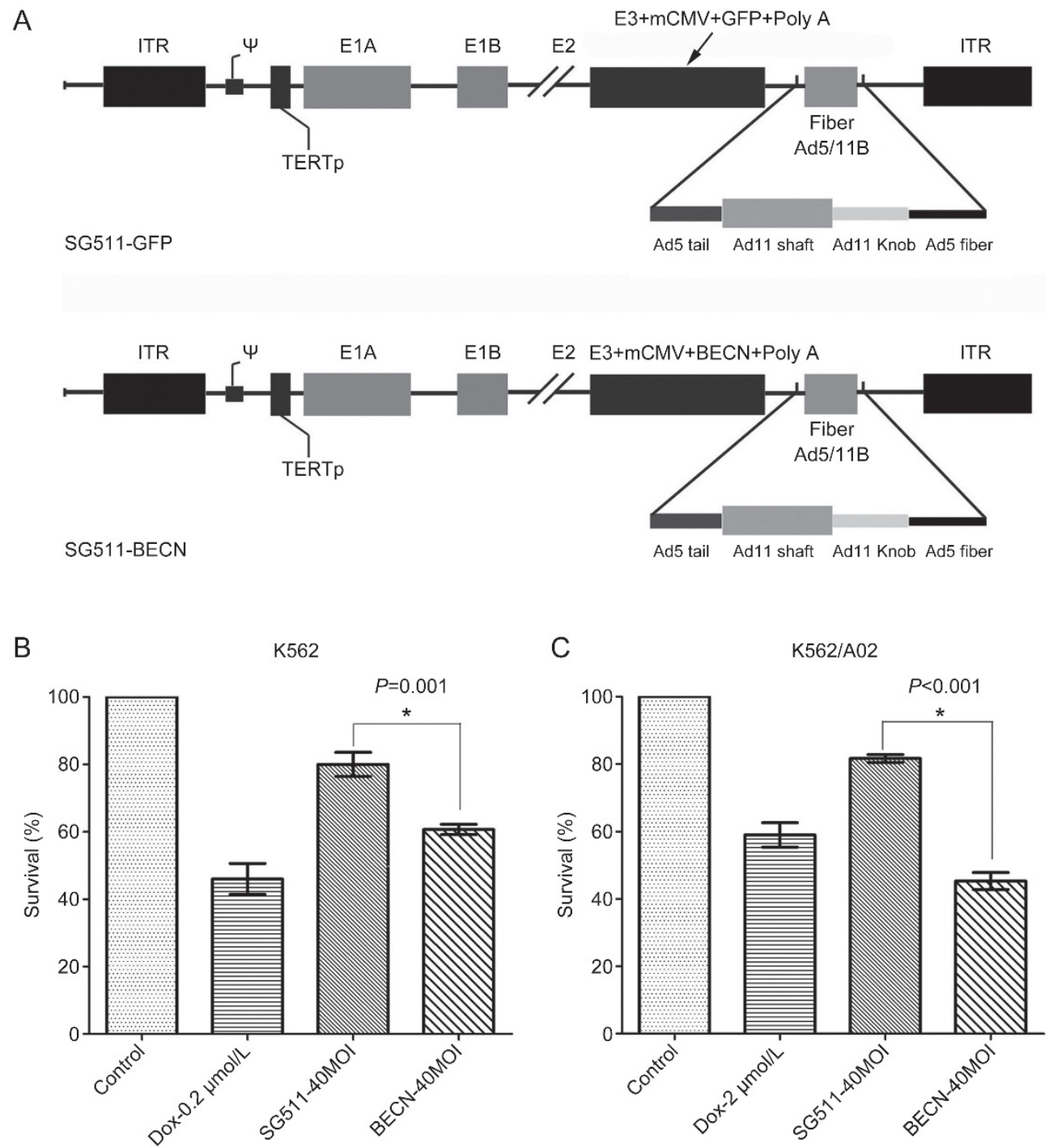

Figure 1. Anticancer activity of the oncolytic virus SG511-BECN to Dox-resistant CML cell lines. Schematic diagram of SG511-GFP and SG511-BECN (A). The oncolytic viruses have an E1B 55-kDa deletion and Ad5/F11 chimeric fiber. Beclin-1 or GFP was cloned into the E3 region. The CML cell line K562 (B) and Dox-resistant CML cell line K562/A02 (C) were exposed to Dox at the indicated concentrations, SG511 (40 MOI), or SG511-BECN (40 MOI) for 48 h. Cell viability was determined by the MTT assay. The results are representative of three independent experiments and are expressed as the mean \pm SD.

SG511-BECN could effectively induce autophagic cell death in chemotherapy-sensitive and -resistant CML cells.

\section{SG511-BECN functions synergistically with Dox to inhibit} multidrug-resistant $\mathrm{CML}$ cell growth

To improve the anti-leukemic efficacy of Dox in CML cells, especially in chemotherapy-resistant cells, we determined whether SG511-BECN enhanced Dox-mediated cell death. The effects of SG511-BECN and Dox alone, as well as SG511BECN plus Dox, on the growth of K562 and K562/ A02 cells were analyzed. K562 and K562/A02 cells were infected with SG511-BECN at an MOI ranging from 5 to 40 in 96-well plates and then were treated with various concentrations of Dox (25-200 nmol/L for K562 cells; 0.25-2 $\mu \mathrm{mol} / \mathrm{L}$ for K562/A02 cells). Cell survival was determined at $72 \mathrm{~h}$ by the MTT assay. As shown in Figure 4A and Figure 4B, cell death induced by SG511-BECN in combination with Dox was significantly increased. CI analysis revealed that the CI for K562 and K562/A02 was <0, suggesting that SG511-BECN and Dox cotreatment was highly synergistic in CML cells. By contrast, SG511-GFP significantly enhanced Dox-mediated cell death in K562 cells (Figure 4C), but not in K562/ A02 cells (Figure 4D).

\section{Mechanism of enhanced cell death induced by the combination} therapy

We investigated the potential molecular mechanism of the sensitization of CML cells to the SG511-BECN/Dox combination by examining possible alterations in the infectious efficiency of SG511-BECN in CML cells when under Dox chemotherapy, and then assessed the expression levels of autophagic and apoptotic signaling molecules.

The leukemic cells were easily transduced by the oncolytic 
A
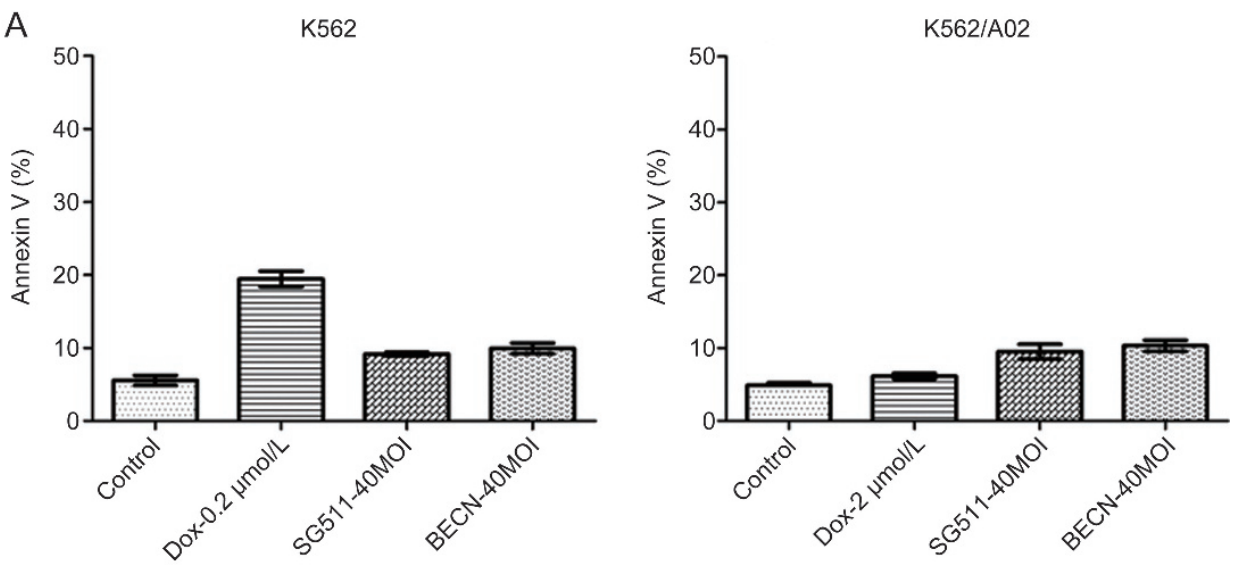

B

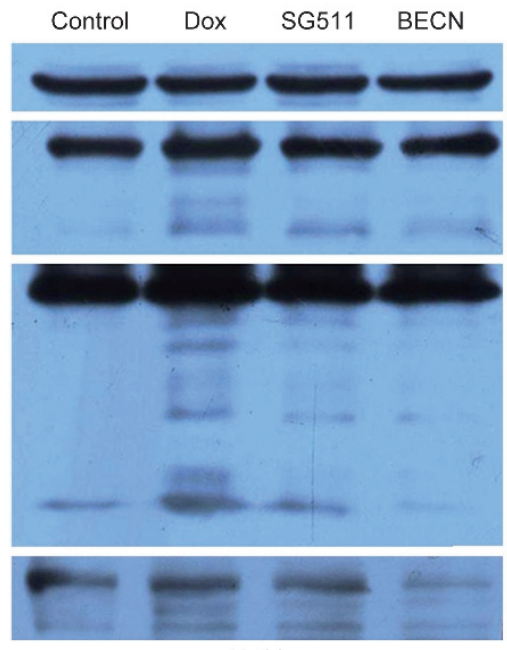

K562

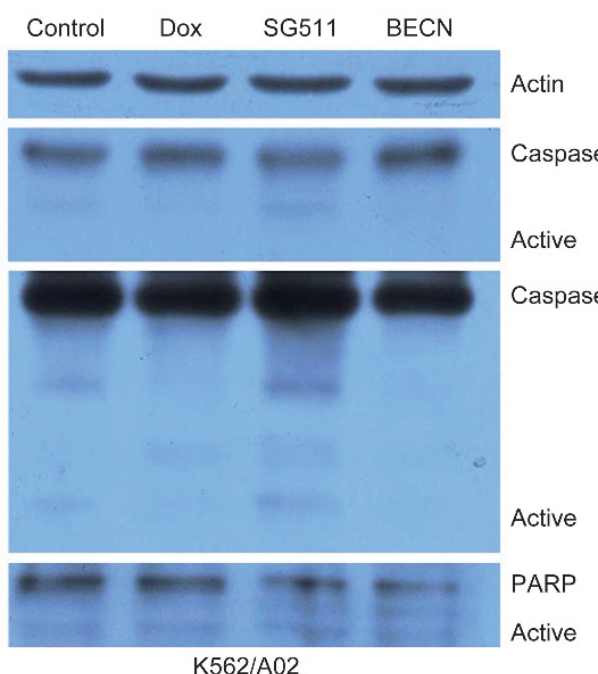

K562/A02

Figure 2. Oncolytic virus SG511-BECN-mediated multidrug-resistant cell-killing effect via an apoptotic-independent way. (A) CML cell lines K562 and K562/A02 were harvested at $48 \mathrm{~h}$ after treatment with $40 \mathrm{MOI}$ of SG511/SG511-BECN virus or different concentrations of Dox. Cells were stained via annexin V/PI staining and were analyzed by flow cytometry. The results are representative of three independent experiments and are expressed as the mean \pm SD. (B) CML cell lines K562 and K562/A02K562 were treated with $40 \mathrm{MOI}$ of SG511/SG511-BECN virus or different concentrations of Dox for $48 \mathrm{~h}$. Whole-cell lysates were subjected to Western blotting analysis to examine the protein levels of caspase-9, caspase-3 and PARP. Actin was used as a loading control. The results are representatives of three independent experiments.

adenovirus, which was described in our previous study ${ }^{[19]}$. As demonstrated in Figure 5A, FACS analysis showed the average portion of GFP-positive cells was obviously increased at 2 days post-infection (MOI=40) in the SG511-GFP/Dox combination group compared with that in the SG511-GFP-alone group, suggesting the infectious efficiency of the oncolytic adenovirus was enhanced when the cancer cells were in the context of chemotherapy.

To further investigate the underlying molecular mechanism by which SG511-BECN functions synergistically with Dox to induce cell death in CML cells, several signaling proteins involved in the autophagic and apoptotic pathway were observed. As shown in Figure 5B and Figure 5C, combined treatment for $48 \mathrm{~h}$ resulted in significantly increased levels of Beclin-1 and LC3-II proteins, while the levels of apoptotic pathway protein expression (caspase-9, -8 and -3) were not altered. These results confirmed that SG511-BECN functions synergistically with Dox to induce cell death in CML cells in an autophagy-targeted manner.

\section{Effect of Dox combined with SG511-BECN on normal human cells}

Next, we examined the effects of Dox combined with SG511BECN on normal human MNCs. The results showed that treating cells with $0.2 \mu \mathrm{mol} / \mathrm{L}$ of Dox or 40 MOI of SG511BECN resulted in $6 \%$ and $8 \%$ cell growth inhibition after 48 $\mathrm{h}$, while the combination of Dox and SG511-BECN resulted in $10 \%$ cell growth inhibition (Figure 5D). Our results suggest that Dox combined with SG511-BECN had limited cytotoxicity to normal human MNCs.

\section{Discussion}

Autophagy is a highly conserved catabolic program for the degradation and recycling of cellular components, including long-lived proteins and organelles, and has been recognized as an important regulatory mechanism in cell fate decisions. Accumulating evidence has indicated that autophagic cell 


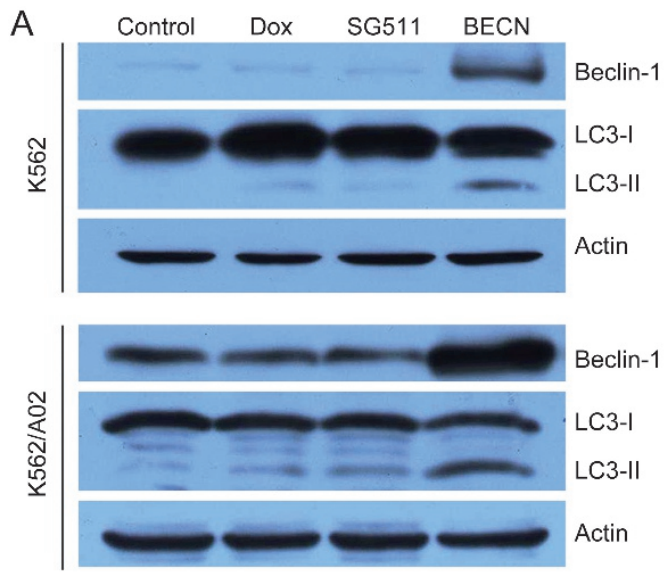

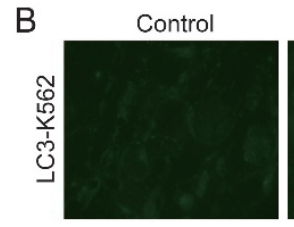

C
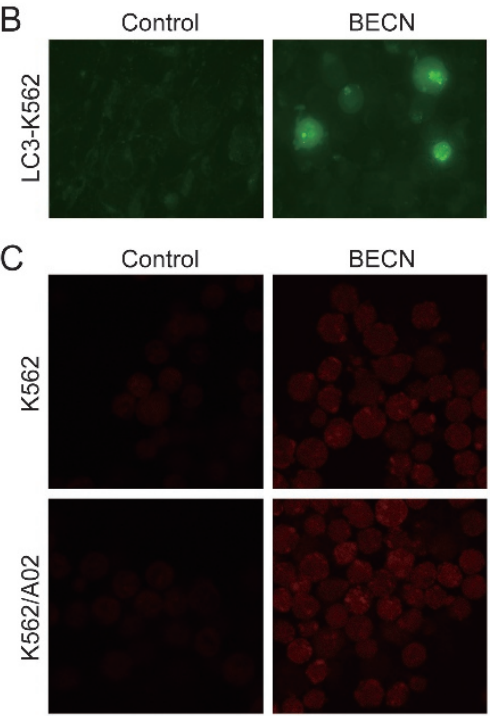

BECN
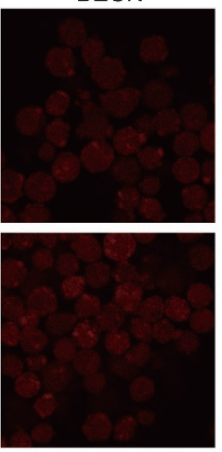

D

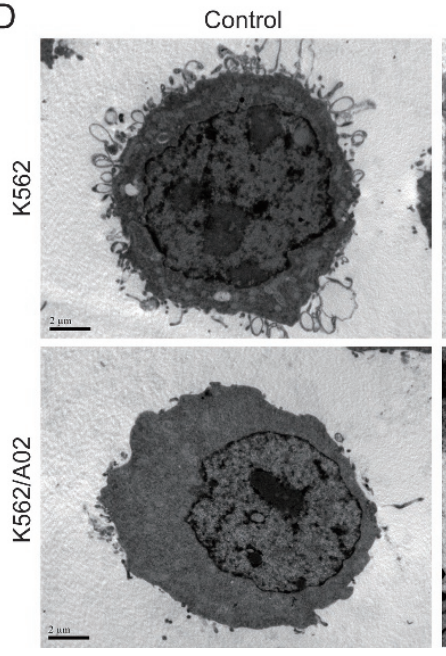

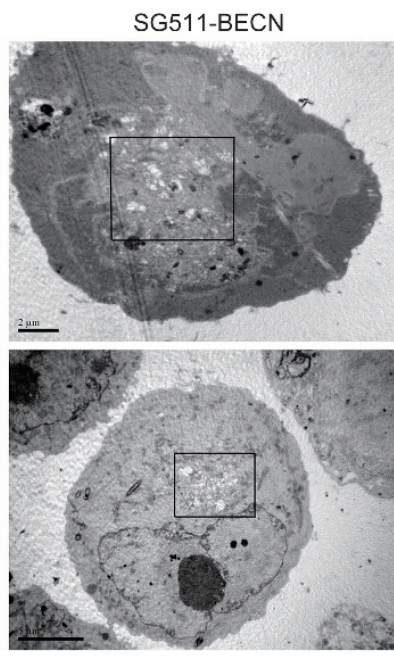

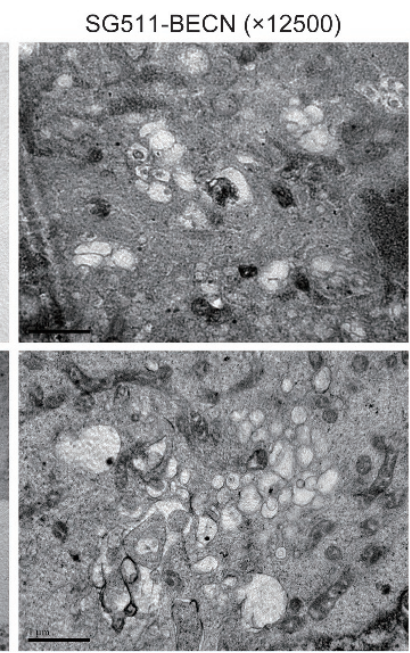

Figure 3. SG511-BECN virus induced autophagic cell death in CML cells. (A) CML cell lines K562 and K562/A02 were infected with the indicated viruses at an $\mathrm{MOI}$ of 40 or different concentrations of Dox $(0.2 \mu \mathrm{mol} / \mathrm{L}$ for $\mathrm{K} 562$ and $2 \mu \mathrm{mol} / \mathrm{L}$ for K562/A02). Cell lysate was immunoblotted with anti-Beclin-1, anti-LC3, and anti-actin antibodies at day 2 of the experiment. (B) Formation of GFP-LC3 vacuoles (dots) was determined after K562 cells were stably transfected with the GFP-LC3 vector and were treated with 40 MOI of SG511 or SG511-BECN for $48 \mathrm{~h}$. (C) Formation of AVOs was determined after CML cells were infected with or without SG511, or SG511-BECN at an MOI of 40 for 48 h. (D) CML cell lines K562 and K562/A02 were treated with SG511 and SG511-BECN, respectively and were observed under TEM ( $\times 4200$ and $\times 12500)$. There are two types of vacuoles in the cytoplasm of SG511-BECN-treated cells: dense multilamellar bodies and inclusion bodies. A representative of two separate experiments is shown.

death plays an important role in the generation of anticancer activity in apoptosis-defective cells ${ }^{[15-17]}$. This process is activated in cancer cells in response to stress or therapeutic agents, which has been implicated as the main mechanism by which some antileukemia agents produce their antileukemic activities $^{[23-26]}$. Interestingly, the induction of autophagy not only mediates cell death induced by dexamethasone in lymphoid leukemia but is also required for leukemic cells to overcome glucocorticoid resistance ${ }^{[23,26]}$. These studies raise the possibility that genes that induce autophagy could act as sensitizers of leukemia cells to the killing effects of oncolytic virus.

In the present study, we investigated whether the antitumor effect of SG511-BECN, a chimeric CRAd armed with Beclin-1, effectively kills human leukemia cell line K562. Additionally, K562/ A02 cells were utilized to examine the effect of SG511BECN on multidrug-resistant cells. The results revealed that the chimeric CRAd plus Beclin-1 achieved superior antileukemia effects compared with the group treated with SG511 virus or Dox alone. Next, we validated the induction of autophagy by our Beclin-1-armed viruses, as indicated by the formation of the autophagic vesicles detected by four independent approaches: LC3-I to LC3-II conversion by Western blotting, LC3 translocation to vacuoles in GFP-LC3-transfected K562 cells, AVO staining and TEM respectively. Consistent with these results, we found that ATG5 and ATG7 knockdown rescued the cells from SG511-BECN-induced growth inhibition 

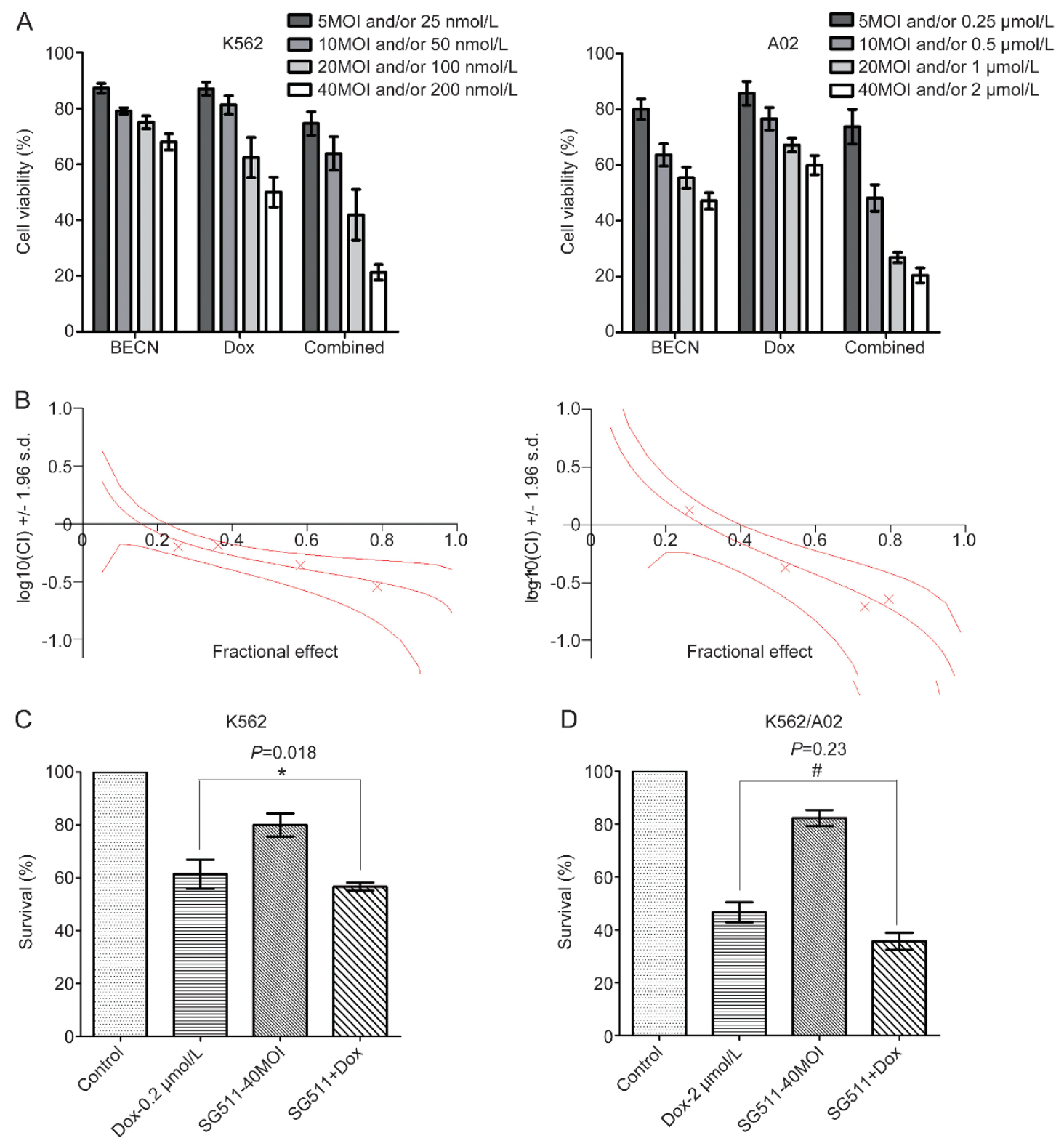

Figure 4. Synergetic cytotoxicity of SG511-BECN or SG511-GFP combined with Dox to inhibit CML cell growth. (A) Dose response of CML cells to SG511BECN, Dox, and SG511-BECN combined with Dox. CML cells were seeded into 96-well plates and were treated with SG511-BECN, Dox alone, or SG511BECN combined with Dox at the dosages indicated. After $72 \mathrm{~h}$, cell viability was measured by the MTT assay. The mean values and standard errors of results from three independent experiments are shown. (B) Synergy was quantified by combination index (Cl) analysis and was expressed as log 10 (Cl) vs fraction affected. Where calculable, 95\% confidence intervals are shown. (C) The CML cell line K562 and (D) Dox-resistant CML cell line K562/A02 were exposed to $40 \mathrm{MOI}$ of SG511-GFP, Dox, or SG511-GFP combined with Dox for $48 \mathrm{~h}$, and then cell viability was determined by the MTT assay. The results are representative of three independent experiments and are expressed as the mean \pm SD.

in our previous studies ${ }^{[19]}$, demonstrating that autophagic cell death contributed to the enhanced cytotoxicity of the virus against leukemia cells.

Several clinical studies have revealed that enhanced and even synergistic antitumor activity can be achieved when oncolytic adenoviruses are used in combination with chemotherapy ${ }^{[27-29]}$ and suggested that the design of CRAd given in combination with cytotoxic chemotherapies will maximize the potential of oncolytic adenovirus and optimize the patient benefit. We previously demonstrated that SG235-TRAIL, a new oncolytic adenovirus harboring an Ad5/F35 chimeric fiber and armed with an antitumor gene TRAIL, acted synergistically with homoharringtonine in human leukemia cell lines ${ }^{[30]}$. In this work, the effects of Dox combined with SG511BECN on CML cells were investigated, and the synergistic effect was identified by the calculation of the CI. The combination therapy was found to significantly inhibit the proliferation of CML cells compared with each agent alone, suggesting that Dox could be used in combination with SG511-BECN in the treatment of leukemia patients. 

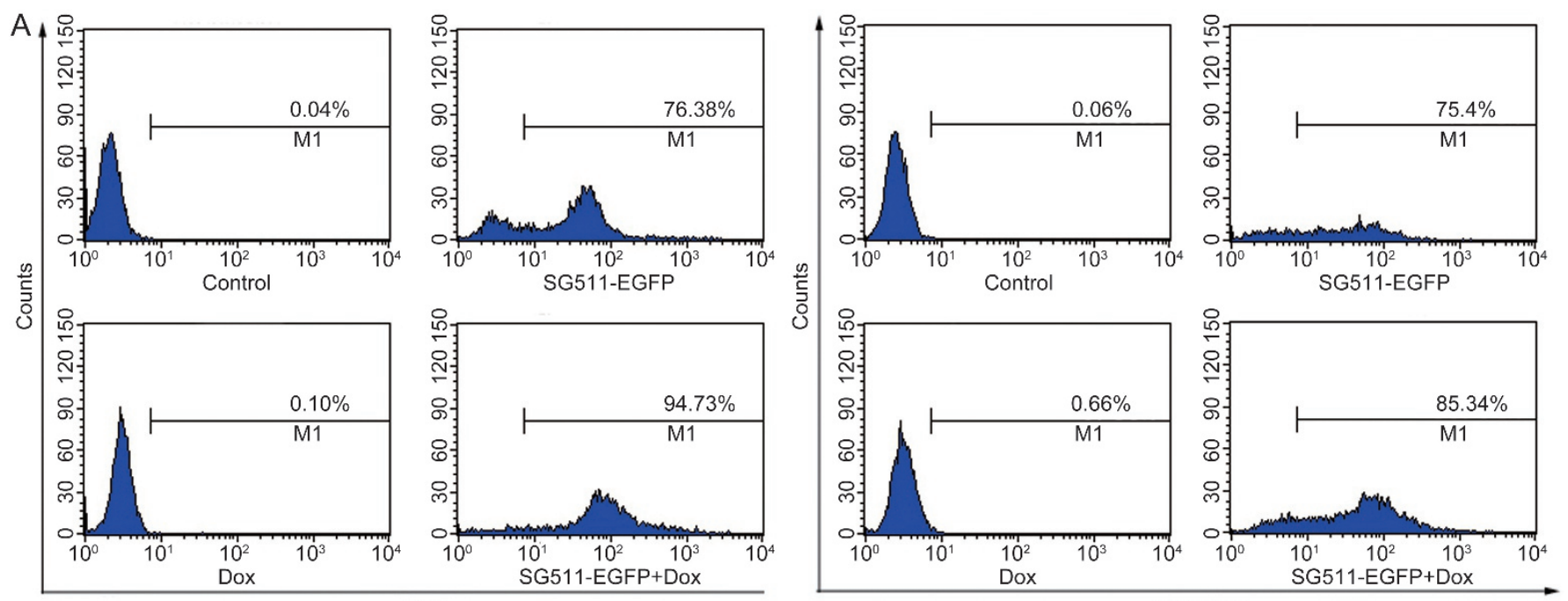

K562

K562/A02

B
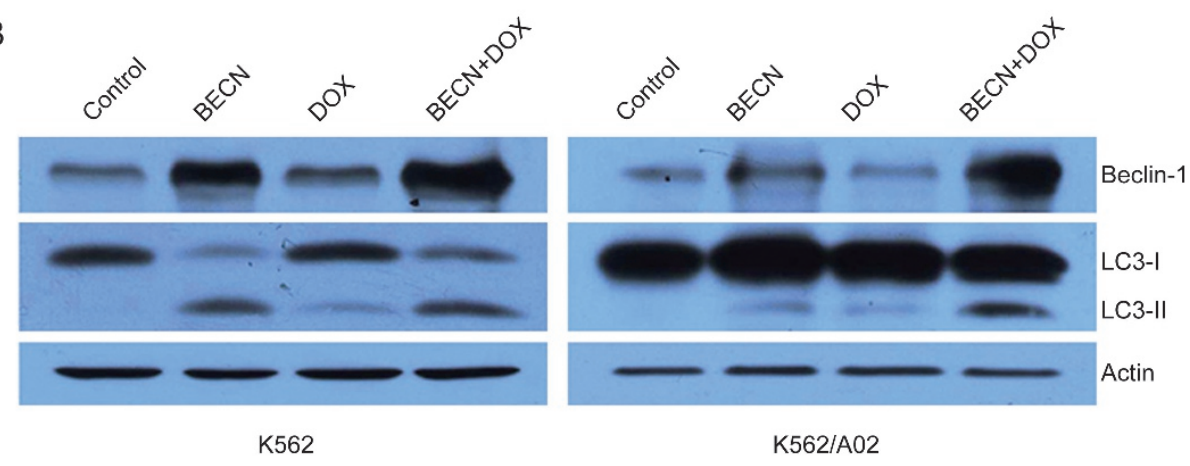

C

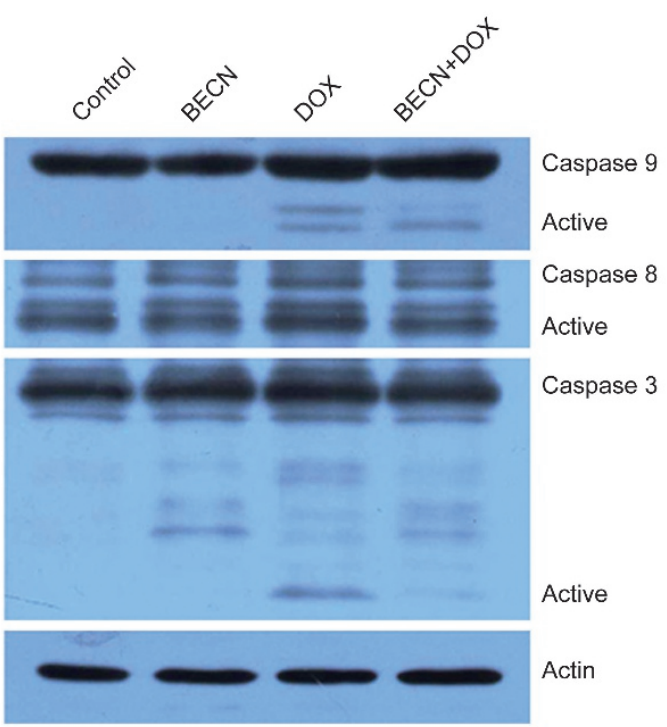

D

MNCS

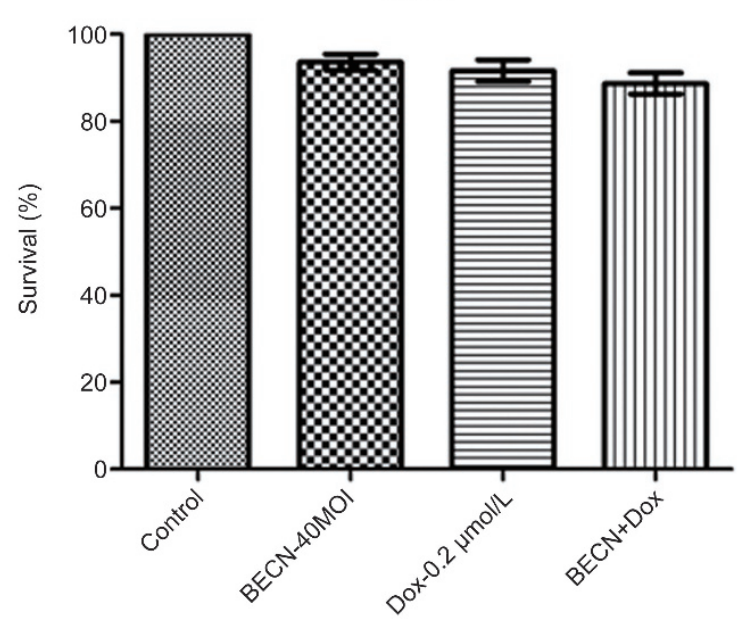

Figure 5. Enhanced infectious efficiency synergizes the autophagic response to combined treatment with Dox and SG511-BECN. (A) K562 and K562/ A02 cells were treated with $40 \mathrm{MOI}$ of SG511-GFP or/and Dox for $24 \mathrm{~h}$ and then collected for analysis by FACS. (B) K562 and K562/A02 cells were infected with the indicated viruses at an $\mathrm{MOI}$ of 40 or different concentrations of Dox (0.2 $\mu \mathrm{mol} / \mathrm{L}$ for K562 and $2 \mu \mathrm{mol} / \mathrm{L}$ for K562/A02). Cell lysates were immunoblotted with anti-Beclin-1, anti-LC3, and anti-actin antibodies at day 2 of the experiment. (C) CML cell lines K562 and K562/A02 cells were treated with $40 \mathrm{MOI}$ of SG511-BECN virus and/or different concentrations of Dox (0.2 $\mu \mathrm{mol} / \mathrm{L}$ for K562 and $2 \mu \mathrm{mol} / \mathrm{L}$ for K562/A02) for $48 \mathrm{~h}$. Whole-cell lysates were subjected to Western blotting analysis to examine the protein levels of caspase-9, caspase-8, caspase-3 and PARP. Actin was used as a loading control. The results are representative of three independent experiments. (D) Normal human mononuclear cells were treated with SG511-BECN virus and/or different concentrations of Dox. Cells that received PBS served as a negative control. After $72 \mathrm{~h}$, cell viability was measured by the MTT assay. The standard error was calculated from three independent experiments. 
To investigate the potential molecular mechanism of the sensitization of CML cells to the SG511-BECN/Dox combination, we detected possible alterations in the infectious efficiency of SG511-BECN in CML cells when under Dox chemotherapy and then observed the expression levels of autophagic/apoptotic signaling molecules. Notably, an interesting finding was that the infectious efficiency of the oncolytic adenovirus was enhanced when the cancer cells were in the context of chemotherapy; however, its underlying mechanism remains vague. The cause might be that chemotherapy agents alter the structure of the cell membrane, making it more susceptible to oncolytic adenovirus infection. The detection of signaling proteins involved in the autophagic and apoptotic pathway confirmed that SG511-BECN functions synergistically with Dox to induce cell death in CML cells in an autophagy-targeted manner.

In summary, we have demonstrated, for the first time, that by introducing the Beclin- 1 gene into the oncolytic adenoviral backbone, the antileukemia activity of the virus on multidrugresistant cell lines can be significantly improved. Furthermore, Dox could synergize with SG511-BECN to kill CML cells by improving the infectious efficiency of the oncolytic adenovirus without causing significant damage to normal human mononuclear cells. These data suggest that expressing the Beclin-1 gene may offer a novel promising gene-virotherapy for the treatment of CML with chemotherapy resistance via a unique mechanism of action distinct from apoptosis, and the combination therapy with chemotherapy agents and oncolytic adenovirus could represent a rational approach for the treatment of CML.

\section{Acknowledgements}

This study was supported by the National Natural Science Foundation of China (№ 81500110), Department of Education of Zhejiang Province (№ Y201120523), Zhejiang Provincial Natural Science Foundation (№ LY15H080001) and Special Scientific Construction Research Funds of National Chinese Medicine Clinical Research Center, SATCM (№ JDZX2015113).

\section{Author contribution}

Wen-bin QIAN designed research; Liang-shun YOU and Li LI performed research; Liang-shun YOU, Li-ping MAO, Shen-he JIN and Xiao-hui CHEN contributed new analytical tools and reagents; Liang-shun YOU analyzed data; Liang-shun YOU wrote the paper.

\section{References}

1 Eager RM, Nemunaitis J. Clinical development directions in oncolytic viral therapy. Cancer Gene Ther 2011; 18: 305-17.

2 Zhang Q, Chen G, Peng L, Wang X, Yang Y, Liu C, et al. Increased safety with preserved antitumoral efficacy on hepatocellular carcinoma with dual-regulated oncolytic adenovirus. Clin Cancer Res 2006; 12: 6523-31.

3 Jin H, Lu S, Yang J, Wang X, Hu H, Su C, et al. Use of microRNA Let7 to control the replication specificity of oncolytic adenovirus in hepatocellular carcinoma cells. PLoS One 2011; 6: e21307.

4 Vasey PA, Shulman LN, Campos S, Davis J, Gore M, Johnston S, et al.
Phase I trial of intraperitoneal injection of the E1B-55-kd-gene-deleted adenovirus ONYX-015 (dl1520) given on days 1 through 5 every 3 weeks in patients with recurrent/refractory epithelial ovarian cancer. J Clin Oncol 2002; 20: 1562-9.

5 Kimball KJ, Preuss MA, Barnes MN, Wang M, Siegal GP, Wan W, et al. A phase I study of a tropism-modified conditionally replicative adenovirus for recurrent malignant gynecologic diseases. Clin Cancer Res 2010; 16: 5277-87.

6 Short JJ, Curiel DT. Oncolytic adenoviruses targeted to cancer stem cells. Mol Cancer Ther 2009; 8: 2096-02.

7 Rivera AA, Davydova J, Schierer S, Wang M, Krasnykh V, Yamamoto M, et al. Combining high selectivity of replication with fiber chimerism for effective adenoviral oncolysis of CAR-negative melanoma cells. Gene Ther 2004; 11: 1694-702.

8 Ranki T, Hemminki A. Serotype chimeric human adenoviruses for cancer gene therapy. Viruses 2010; 2: 2196-212.

9 Hermiston T. Gene delivery from replication-selective viruses: arming guided missiles in the war against cancer. J Clin Invest 2000; 105: 1169-72.

10 Liu XY, Qiu SB, Zou WG, Pei ZF, Gu JF, Luo CX, et al. Effective genevirotherapy for complete eradication of tumor mediated by the combination of hTRAIL (TNFSF10) and plasminogen k5. Mol Ther 2005; 11: 531-41.

11 He X, Liu J, Yang C, Su C, Zhou C, Zhang Q, et al. 5/35 fiber-modified conditionally replicative adenovirus armed with p53 shows increased tumor-suppressing capacity to breast cancer cells. Hum Gene Ther 2011; 22: 283-92.

12 Yang C, Tong Y, Ni W, Liu J, Xu W, Li L, et al. Inhibition of autophagy induced by overexpression of mda-7/interleukin-24 strongly augments the antileukemia activity in vitro and in vivo. Cancer Gene Ther 2010; 17: 109-19.

13 Mashima T, Tsuruo T. Defects of the apoptotic pathway as therapeutic target against cancer. Drug Resist Updat 2005; 8: 339-43.

14 Chen N, Karantza V. Autophagy as a therapeutic target in cancer. Cancer Biol Ther 2011; 11: 157-68.

15 Chen Y, McMillan-Ward E, Kong J, Israels SJ, Gibson SB. Oxidative stress induces autophagic cell death independent of apoptosis in transformed and cancer cells. Cell Death Differ 2008; 15: 171-82.

16 Høyer-Hansen M, Bastholm L, Mathiasen IS, Elling F, Jäättelä M. Vitamin D analog EB1089 triggers dramatic lysosomal changes and Beclin 1-mediated autophagic cell death. Cell Death Differ 2005; 12 : 1297-309.

17 Qian W, Liu J, Jin J, Ni W, Xu W. Arsenic trioxide induces not only apoptosis but also autophagic cell death in leukemia cell lines via upregulation of Beclin-1. Leuk Res 2007; 31: 329-39.

18 Carew JS, Nawrocki ST, Kahue CN, Zhang H, Yang C, Chung L, et al. Targeting autophagy augments the anticancer activity of the histone deacetylase inhibitor SAHA to overcome Bcr-Abl-mediated drug resistance. Blood 2007; 110: 313-22.

19 Tong Y, You L, Liu H, Li L, Meng H, Qian Q, et al. Potent antitumor activity of oncolytic adenovirus expressing Beclin-1 via induction of autophagic cell death in leukemia. Oncotarget 2013; 4: 860-74.

20 Kimura S, Ando T, Kojima K. Ever-advancing chronic myeloid leukemia treatment. Int J Clin Oncol 2014; 19: 3-9.

21 Hochhaus A, O'Brien SG, Guilhot F, Druker BJ, Branford S, Foroni L, et al. Six-year follow-up of patients receiving imatinib for the first-line treatment of chronic myeloid leukemia. Leukemia 2009; 23: 105461.

22 Mahon FX, Deininger MW, Schultheis B, Chabrol J, Reiffers J, Goldman $\mathrm{JM}$, et al. Selection and characterization of BCR-ABL positive cell lines with differential sensitivity to the tyrosine kinase inhibitor STI571: 
diverse mechanisms of resistance. Blood 2000; 96: 1070-9.

23 Laane E, Tamm KP, Buentke E, Ito K, Kharaziha P, Oscarsson J, et al. Cell death induced by dexamethasone in lymphoid leukemia is mediated through initiation of autophagy. Cell Death Differ 2009; 16 : 1018-29.

24 Goussetis DJ, Altman JK, Glaser H, McNeer JL, Tallman MS, Platanias LC. Autophagy is a critical mechanism for the induction of the antileukemic effects of arsenic trioxide. J Biol Chem 2010; 285: 29989-97.

25 Heidari N, Hicks MA, Harada H. GX15-070 (obatoclax) overcomes glucocorticoid resistance in acute lymphoblastic leukemia through induction of apoptosis and autophagy. Cell Death Dis 2010; 1: e76.

26 Bonapace L, Bornhauser BC, Schmitz M, Cario G, Ziegler U, Niggli $\mathrm{FK}$, et al. Induction of autophagy-dependent necroptosis is required for childhood acute lymphoblastic leukemia cells to overcome glucocorticoid resistance. J Clin Invest 2010; 120: 1310-23.

27 Kruyt FA, Curiel DT. Toward a new generation of conditionally replicating adenoviruses: pairing tumor selectivity with maximal oncolysis. Hum Gene Ther 2002; 13: 485-95.

28 Post DE, Khuri FR, Simons JW, Van Meir EG. Replicative oncolytic adenoviruses in multimodal cancer regimens. Hum Gene Ther 2003; 14: 933-46.

29 Libertini S, lacuzzo I, Ferraro A, Vitale M, Bifulco M, Fusco A, et al. Lovastatin enhances the replication of the oncolytic adenovirus dl1520 and its antineoplastic activity against anaplastic thyroidcarcinoma cells. Endocrinology 2007; 148: 5186-94.

30 Meng HT, Li L, Liu H, Wang Y, Li GC, Qian W. Homoharringtonine acts synergistically with SG235-TRAIL, a conditionally replicating adenovirus, in the treatment of leukemia. Acta Pharmacol Sin 2009; 11: $1529-36$. 